\title{
Spin-resolved neutron spectroscopy from the heavy Fermion compound $\mathrm{CeCu}_{6}$
}

\author{
T.J. Hicks ${ }^{\mathrm{a}}$, D.J. Goossens ${ }^{\mathrm{b}, 1}$, S.J. Harker ${ }^{\mathrm{a}, *}$, A.M. Mulders ${ }^{\mathrm{a}, 2}$, S.J. Kennedy ${ }^{\mathrm{b}}$ \\ ${ }^{a}$ School of Physics and Materials Engineering, Monash University, PO Box 27, Vic 3800, Australia \\ ${ }^{\mathrm{b}}$ Bragg Institute, Australian Nuclear Science and Technology Organisation, PMB 1, Menai, NSW 2234, Australia
}

\begin{abstract}
Neutron time-of-flight spectroscopy with neutron polarisation analysis permits the in situ separation of magnetic and lattice vibrational energy spectra. Preliminary experiments on the heavy Fermion compound, $\mathrm{CeCu}_{6}$, in which the $\mathrm{Ce}$ magnetic moment is suppressed by the Kondo effect, allow an indicative separation of a broadened crystal field transition and features due to lattice vibrations. An inelastic spin-flip feature at $-12 \mathrm{meV}$ is due to the crystal field while an inelastic non-spin-flip feature at $-6 \mathrm{meV}$ is predominantly due to phonon scattering.
\end{abstract}

(C) 2003 Elsevier B.V. All rights reserved.

PACS: 25.40.Fq; 29.27.Hj; 71.70.Ch

Keywords: $\mathrm{CeCu}_{6}$; Crystal field; Neutron polarisation analysis; Time of flight

The heavy Fermion behaviour in $\mathrm{CeCu}_{6}$ is a consequence of the competition between the Ruderman-Kittel-Kasuya-Yoshida (RKKY) interaction and the Kondo effect due to the admixture of the $4 \mathrm{f}$ and conduction electrons. The interaction between the heavy Fermion and crystal field (CF) excitations leads to broad transitions which are further complicated by the presence of phonon scattering over the region of interest. A number of investigations of the $\mathrm{CF}$ level scheme in the heavy fermion compound

\footnotetext{
*Corresponding author. Tel.: +61-3-9905-3632; fax: +61-39905-3637.

E-mail address: stephen.harker@spme.monash.edu.au (S.J. Harker).

${ }^{1}$ Current address: Research School of Chemistry, Australian National University, ACT 0200, Australia.

${ }^{2}$ Current address: Department of Physics, Uppsala University, Box 530, S-75121 Uppsala, Sweden.
}

$\mathrm{CeCu}_{6}$ have been made. These include inelastic neutron scattering (INS) measurements [1-3]. These measurements proposed different CF level transitions. Walter et al. proposed a transition at $5.5 \mathrm{meV}$ from a $50 \mathrm{~K}$ measurement [1]. Goremychkin and Osborn found possible transitions at 0, 7.0 and $13.8 \mathrm{meV}$ from a $20 \mathrm{~K}$ measurement [2]. Stroka et al. reported possible transitions at 7-8 and $12-14 \mathrm{meV}$ from a $3 \mathrm{~K}$ measurement.

Conventional INS spectra without polarisation analysis (PA) require an additional spectrum of an equivalent system with a non-magnetic rare earth to distinguish between magnetic and lattice spectral features. Neutron time of flight spectroscopy with PA analysis permits the in situ separation of magnetic and lattice vibrational energy spectra [4]. In this work we report on preliminary measurements of this type performed on $\mathrm{CeCu}_{6}$. 
Above $230 \mathrm{~K}, \mathrm{CeCu}_{6}$ has an orthorhombic Pnma structure in which the $\mathrm{Ce}$ atoms are located at $4 \mathrm{c}$ sites having monoclinic $\mathrm{m}\left(\mathrm{C}_{\mathrm{s}}\right)$ local symmetry [5]. Below $230 \mathrm{~K}$ a slight monoclinic distortion takes effect and $\mathrm{CeCu}_{6}$ has the monoclinic $\mathrm{P} 2_{1} / \mathrm{c}$ structure with the $\mathrm{Ce}$ atoms located at 4e sites having triclinic $1\left(\mathrm{C}_{1}\right)$ local symmetry [6]. The $\mathrm{Ce}^{3+}$ ion has a ${ }^{2} \mathrm{~F}_{5 / 2}$ ground state which is split into three Kramer's doublets by the crystal field due to the surrounding ions and hence there are three transitions between the CF split levels.

A polycrystalline $\mathrm{CeCu}_{6}$ sample was prepared by argon-arc melting of stoichiometric amounts of high purity $\mathrm{Ce}$ and $\mathrm{Cu}$. The ingot was annealed for $120 \mathrm{~h}$ at $900^{\circ} \mathrm{C}$ in flowing argon. The annealed sample was analysed by neutron diffraction at ANSTO. A Rietveld refinement showed the composition to be very close to pure, with at most a few atomic percent of $\mathrm{Cu}$ metal as impurity. As $\mathrm{Cu}$ will not contribute to the $\mathrm{CF}$ spectrum, this was deemed quite acceptable.

Neutron time-of-flight spectra were taken on LONGPOL, at $26 \mathrm{~K}$ with an incident wavelength of $3.6 \AA(6.3 \mathrm{meV})$, using the polycrystalline sample. The neutron polarisation direction was along the elastic scattering vector of the middle of eight detectors which spanned an angle of $50^{\circ}(0.3-$ $\left.1.75 \AA^{-1}\right)$. The polarisation of the beam was statistically chopped and the spectrum reconstructed by cross-correlation of the flipping sequence with the time sequence of the intensity in the detectors [7]. The flipping sequence was a shift register sequence of 31 elements and the ratio between flipper on and flipper off was 15/16. Shift register sequences have an autocorrelation function which is a single triangle at zero shift. This is illustrated schematically in Fig. 1.

In the configuration with neutron polarisation along the scattering vector the magnetic excitations scatter with neutron spin-flip (SF). Insofar as all the detectors are not at the inelastic scattering vector and that the inelastic scattering vector deviates from the elastic scattering vector most, but not all, of the magnetic scattering is with neutron SF. The lattice vibrations, however scatter without neutron SF. The reconstructed spectrum is proportional to the non-spin-flip (NSF) minus SF intensity [4] as is illustrated in Fig. 1. As a (a)

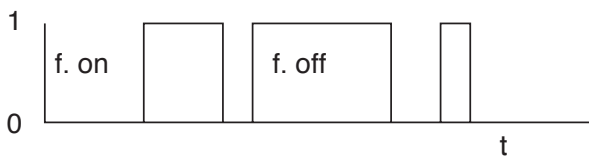

(b)

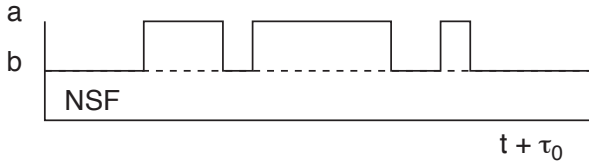

(c)

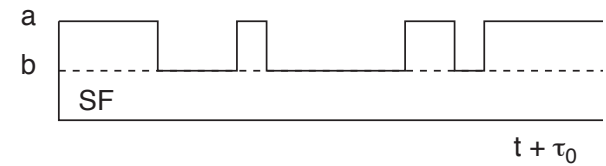

(d)

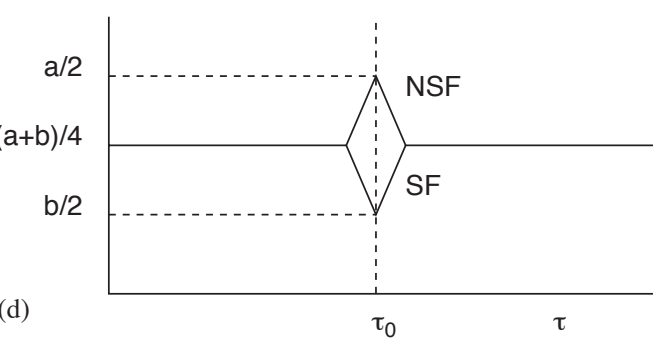

Fig. 1. Schematic explanation of the random or pseudorandom time of flight system: (a) part of the pulse sequence sent to the spin flipper; (b), (c) the resulting variations in the non-spin-flip (NSF) and spin-flip (SF) scattering intensities at the detector. (d) Also shown are simple time-of-flight spectra resulting from the cross-correlation of impressed and resulting time sequences.

result the magnetic scattering is largely discriminated from nuclear scattering for which there is no change in polarisation. This is important as the nuclear inelastic scattering dominates the spectrum of $\mathrm{CeCu}_{6}$ [2].

The spectrum obtained at $26 \mathrm{~K}$ summed over all detectors except one is shown in Fig. 2. One detector is excluded as it contained a large Bragg peak. With an incident energy of $6.3 \mathrm{meV}$, the usable data was mostly with neutron energy gain. The data shows a large nuclear (NSF) elastic peak, a broad nuclear (NSF) feature around $-6 \mathrm{meV}$ and two magnetic (SF) features at -2 and $-12 \mathrm{meV}$.

The magnetic feature near $-2 \mathrm{meV}$ is the wing of the quasielastic peak first seen by Walter et al. This is supported by the observation of a similar 


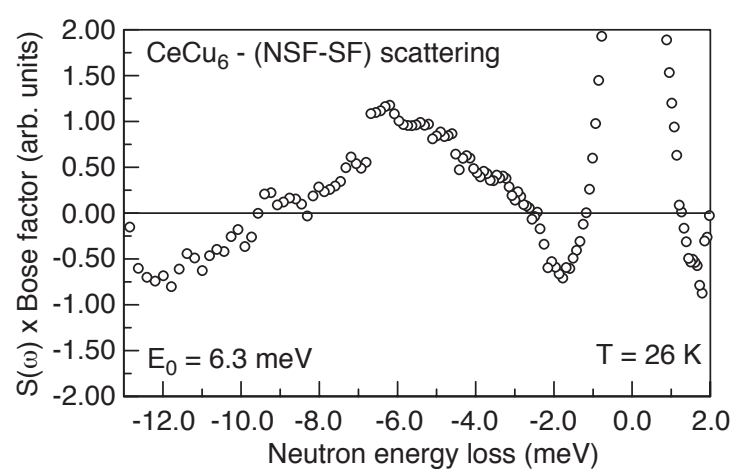

Fig. 2. Neutron time-of-flight energy spectrum obtained from $\mathrm{CeCu}_{6}$ at $26 \mathrm{~K}$ as measured on LONGPOL. The crystal field (magnetic) scattering appears as dips or negative going peaks, lattice scattering appears as positive peaks.

feature of similar energy on the neutron energy loss (right-hand side) of the elastic position, suggesting that the nuclear elastic scattering is situated within a broader magnetic quasielastic dip. The magnetic feature at $-12 \mathrm{meV}$ is consistent with a CF transition identified by both Goremychkin and Osborn and by Stroka et al., although the full extent of this very broad transition has not been mapped, nor have higher energy transitions been ruled out. An inelastic scattering event at $-6 \mathrm{meV}$ has been identified as a CF transition by all previous studies. However, Fig. 2 shows a positive cross-correlation at this energy, indicating predominantly NSF scattering which cannot be due to a $\mathrm{CF}$ transition. This demonstrates the key strength of the PA experiment.

The spectrum in Fig. 2 is proportional to the difference between NSF and SF scattering. However the spectral information is superimposed on a relatively large uncorrelated background related to all the scattering. Definitive separation of NSF and SF events can be achieved by switching the neutron polarisation direction be- tween the present geometry and one with polarisation perpendicular to the scattering plane. In the latter geometry the spectral information contains only NSF scattering and a subtraction of the two sets of data not only removes the uncorrelated background but isolates the magnetic scattering. The present spectra can only reveal the predominant mode of scattering at each energy transfer. Definitive separation must await an experiment in which the polarisation direction is switched. This is being pursued.

Further data will be taken at higher temperatures so that higher levels can be populated and further possible crystal field transitions seen. The transitions are broadened at higher temperatures and this might limit their visibility. We also plan to examine samples in which one $\mathrm{Cu}$ site is substituted with $\mathrm{Au}$. This weakens the Kondo interaction and sharpens the CF levels [3].

\section{Acknowledgements}

We acknowledge the support of the Australian Research Council and of the Australian Institute of Nuclear Science and Engineering.

\section{References}

[1] U. Walter, D. Wohlleben, Z. Fisk, Z. Phys. B 62 (1986) 325.

[2] E.A. Goremychkin, R. Osborn, Phys. Rev. B 47 (21) (1993) 14580.

[3] B. Stroka, A. Schröder, T. Trappmann, H.V. Löhneysen, M. Loewenhaupt, A. Severing, Z. Phys. B 90 (1993) 155.

[4] D.J. Goossens, S.J. Kennedy, T.J. Hicks, Nucl. Instrum. Methods A 380 (1996) 572.

[5] D.T. Cromer, A.C. Larson, R.B. Roof Jr., Acta Crystallogr. 13 (1960) 913.

[6] M.L. Vrtis, J.D. Jorgensen, D.G. Hinks, Physica B 136 (1986) 489.

[7] T.J. Hicks, Adv. Phys. 45 (4) (1996) 243. 